\title{
Segment length in cine (SLICE) strain analysis: a practical approach to estimate potential benefit from cardiac resynchronization therapy
}

\author{
Alwin Zweerink ${ }^{1}$, Robin Nijveldt ${ }^{1,2}$, Natalia J. Braams ${ }^{1}$, Alexander H. Maass ${ }^{3}$, Kevin Vernooy ${ }^{2,4}$, \\ Frederik J. de Lange ${ }^{5}$, Mathias Meine ${ }^{6}$, Bastiaan Geelhoed ${ }^{3}$, Michiel Rienstra ${ }^{3}$, Isabelle C. van Gelder ${ }^{3}$, \\ Marc A. Vos ${ }^{7}$, Albert C. van Rossum ${ }^{1}$ and Cornelis P. Allaart ${ }^{*}$
}

\begin{abstract}
Background: Segment length in cine (SLICE) strain analysis on standard cardiovascular magnetic resonance (CMR) cine images was recently validated against gold standard myocardial tagging. The present study aims to explore predictive value of SLICE for cardiac resynchronization therapy (CRT) response.

Methods and results: Fifty-seven patients with heart failure and left bundle branch block (LBBB) were prospectively enrolled in this multi-center study and underwent CMR examination before CRT implantation. Circumferential strains of the septal and lateral wall were measured by SLICE on short-axis cine images. In addition, timing and strain pattern parameters were assessed. After twelve months, CRT response was quantified by the echocardiographic change in left ventricular (LV) end-systolic volume (LVESV). In contrast to timing parameters, strain pattern parameters being systolic rebound stretch of the septum $\left(\mathrm{SRS}_{\text {sep }}\right)$, systolic stretch index (SSI $\mathrm{sep}_{\text {-lat }}$ ), and internal stretch factor (ISF sep-lat $_{\text {f }}$ ) all correlated significantly with LVESV change $(R-0.56 ; R-0.53$; and $R-0.58$, respectively). Of all strain parameters, end-systolic septal strain $\left(E_{S S}\right.$ sep $)$ showed strongest correlation with LVESV change $(R-0.63)$. Multivariable analysis showed $E_{\text {SS }}$ to be independently related to LVESV change together with age and QRS AREA.

Conclusion: The practicable SLICE strain technique may help the clinician to estimate potential benefit from CRT by analyzing standard CMR cine images without the need for commercial software. Of all strain parameters, end-systolic septal strain $\left(E_{S S}\right.$ sep $)$ demonstrates the strongest correlation with reverse remodeling after CRT. This parameter may be of special interest in patients with non-strict LBBB morphology for whom CRT benefit is doubted.
\end{abstract}

Keywords: Cardiovascular magnetic resonance (CMR), Segment length in cine (SLICE), Myocardial strain, Cardiac resynchronization therapy (CRT)

\footnotetext{
*Correspondence: cp.allaart@amsterdamumc.nl

${ }^{1}$ Department of Cardiology, Amsterdam Cardiovascular Sciences (ACS),

Amsterdam University Medical Centers (AUMC), Location VU University Medical Center, De Boelelaan 1118, 1081 HV Amsterdam, The Netherlands

Full list of author information is available at the end of the article
}

\section{Introduction}

Cardiac resynchronization therapy (CRT) is an established therapy for patients with chronic heart failure, reduced left ventricular (LV) ejection fraction and left bundle branch block (LBBB). Patient selection is primarily guided by the electrocardiogram (ECG), but this results in approximately one-third of patients not benefitting from the therapy $[1,2]$. Additional criteria are original author(s) and the source, provide a link to the Creative Commons licence, and indicate if changes were made. The images or other third party material in this article are included in the article's Creative Commons licence, unless indicated otherwise in a credit line to the material. If material is not included in the article's Creative Commons licence and your intended use is not permitted by statutory regulation or exceeds the permitted use, you will need to obtain permission directly from the copyright holder. To view a copy of this licence, visit http://creativecommons.org/licenses/by/4.0/. The Creative Commons Public Domain Dedication waiver (http://creativeco mmons.org/publicdomain/zero/1.0/) applies to the data made available in this article, unless otherwise stated in a credit line to the data. 
therefore needed to improve patient selection for CRT. Myocardial strain imaging provides mechanical information on LV regional timing differences (also known as dyssynchrony) and inefficient contraction patterns (also known as discoordination). Strain parameters have been linked to CRT outcome in prior studies [3-6]. At present, multiple strain imaging tools are commercially available for both cardiovascular magnetic resonance (CMR) and echocardiographic modalities $[7,8]$. CMR strain analysis requires additional tagging sequences (CMR-TAG), or can be performed on standard cine images using specialized feature tracking (CMR-FT) software. In addition, we recently validated the novel segment length in cine (SLICE) technique. The purpose of SLICE is to provide the clinician a simple and transparent method to estimate benefit from CRT. More specifically, SLICE consists of a series of manual frame-to-frame segment length measurements between anatomic landmarks on standard short-axis CMR cines [9]. This technique showed excellent intra-observer and good inter-observer reproducibility for measuring circumferential strains. Moreover, SLICE showed good agreement with gold standard CMRTAG and holds the advantage of being widely available as it requires no additional image acquisition sequences (i.e. tagging), or commercial post-processing software (i.e. feature tracking). The aim of the present study was to evaluate SLICE as a practicable strain technique to estimate potential benefit from CRT using standard CMR cine images.

\section{Methods}

\section{Study population}

This study was part of the Markers And Response to CRT (MARC) study, a non-randomized, multi-center study that investigated the role of various clinical-, ECG-, echocardiographic-, and biomarker parameters to predict LV reverse remodeling after CRT [10]. The MARC study prospectively enrolled two-hundred-forty patients planned for CRT implantation in six medical centers in the Netherlands. In the present study, 57 patients from five participating centers were included who underwent CMR examination in three of the following experienced centers (Maastricht University Medical Centre, University Medical Centre Groningen and Amsterdam University Medical Centers, location VU medical center; the latter also performing CMR studies for two other participating centers). Previously, we validated SLICE against CMR-TAG in 27 patients who underwent CMR examination with additional CMR-TAG imaging in our center [9]. All subjects gave written informed consent and the local medical ethics committee (Amsterdam University Medical Centers, location VU medical center) approved data collection and management. The investigation conforms with the principles outlined in the Declaration of Helsinki.

\section{Definition of CRT response}

Device programming was DDD mode in all patients with a sensed atrioventricular (AV) delay of $90 \mathrm{~ms}$, paced AVdelay of $130 \mathrm{~ms}$ and interventricular (VV) delay $0 \mathrm{~ms}$. Echocardiographic assessment of LV volumes was performed before, and twelve months after CRT implantation. LV end-systolic volume (LVESV) was measured using the biplane Simpson's method by two experienced observers. Patients with $\geq 15 \%$ reduction in LVESV were classified as CRT responders [11].

\section{Image acquisition}

Patients underwent CMR examination using a $1.5 \mathrm{~T}$ system (Magnetom Avanto or Aera, Siemens Healthineers, Erlangen, Germany; or Intera CV, Philips Healthcare, Best, The Netherlands). Standard CMR cine images were acquired using a retrospectively ECG-gated balanced steady-state free-precession (bSSFP) sequence with standard short-axis and long-axis orientations to measure LV volumes and calculate LV ejection fraction (LVEF). Typically, temporal resolution was $<50 \mathrm{~ms}$ and the number of reconstructed temporal phases within the cardiac cycle was set between 20 and 30. Typical image acquisition parameters were: slice thickness/slice gap: $5 / 5 \mathrm{~mm}$, $8 / 0 \mathrm{~mm}$ or $6 / 4 \mathrm{~mm}$; echo time (TE)/repetition time (TR): $1.6 \mathrm{~ms} / 3.2 \mathrm{~ms}$; in-plane spatial resolution: 1.5 by $2.1 \mathrm{~mm}$; flip angle: 45 to 75 degrees. Cine imaging of the LV in the three-chamber view was performed to assess aortic valve closure (AVC). Myocardial scar territory was assessed by late gadolinium enhancement (LGE) imaging, and infarct size was measured using the full width at half maximum method [12]. All CMR data were analyzed using dedicated offline software (QMassMR version 7.6, Medis, Leiden, The Netherlands).

\section{SLICE strain analysis}

A detailed description of the SLICE analysis post-processing steps has been published previously [9]. In brief, the mid-LV slice position with short-axis cine images was selected (QMassMR version 7.6, Medis). Two endocardial anatomic landmarks (trabeculae) near the right ventricular (RV) insertion points, delimiting the septal segment, were chosen in the end-diastolic (ED) frame. Marks were placed perpendicular to the myocardium following the anatomic landmarks throughout all phases. This was repeated for the lateral wall segment. Localization of lateral wall landmarks was performed by drawing a straight line from the RV insertion points through 


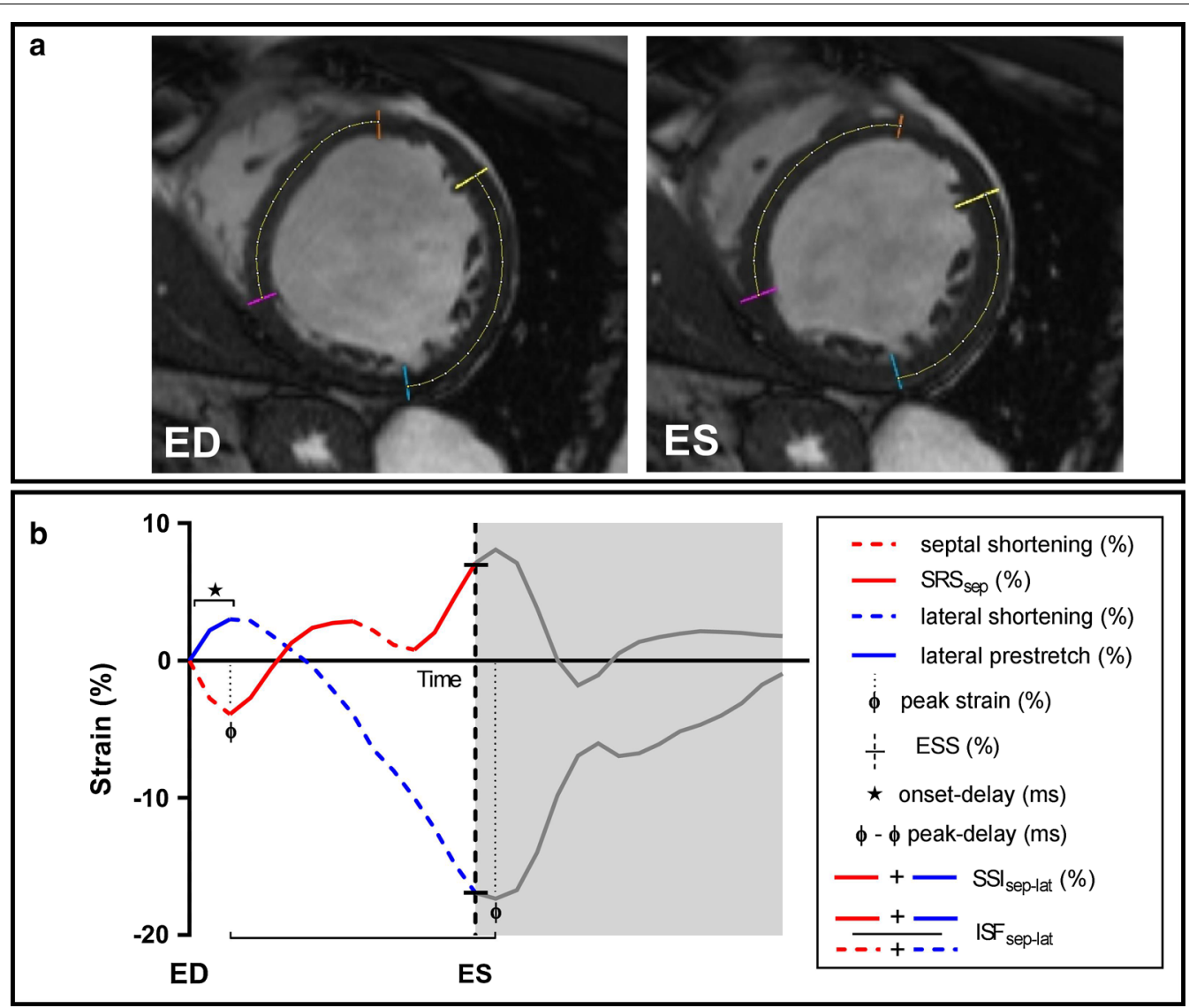

Fig. 1 SLICE analysis and measurement of strain parameters. a Typical example of a left bundle branch block (LBBB) patient with segment length in cine (SLICE) analysis of the septal and lateral wall in the end-diastolic (ED) and end-systolic (ES) frame. $\mathbf{b}$ Strain curves of the septal (red) and lateral (blue) wall with measurement of basic strain values as the maximal negative strain value (peak strain) and end-systolic strain value (ESS) at aortic valve closure (determined by cine imaging of the left ventricle (LV) in the three-chamber view); timing differences measured as the septal to lateral delay in onset contraction (onset-delay) and peak negative strain delay (peak-delay); and inefficient strain patterns measured as systolic rebound stretch of the septum $\left(\mathrm{SRS}_{\text {sep }}\right)$, systolic stretch index $\left(\mathrm{SSI}_{\text {sep-lat }}\right)$ and internal stretch index $\left(\mathrm{ISF}_{\text {sep-lat }}\right)$

the LV center point to the opposite site (see Additional file 1: Figure S1). Subsequently, marked cine images were exported to ImageJ to measure segment length measurements between the marks over the myocardium midline in each phase using the segmented line tool, see Fig. 1a. Segments lengths were expressed as a percentage of the ED segment length, and the frame-to-frame segment length change was plotted as a strain curve, see Fig. 1b.

\section{Strain parameters}

Four subsets of strain parameters were evaluated. First, basic strain values were quantified by the septal and lateral peak negative strain (peak strain); and end-systolic strain (ESS) at the time of AVC (determined by cine imaging of the LV in the three-chamber view). Secondly, timing differences were measured by the septalto-lateral delay in onset of shortening (onset-delay); and the septal-to-lateral time difference in peak contraction (peak-delay); Thirdly, strain pattern parameters included systolic rebound stretch of the septum $\left(\mathrm{SRS}_{\mathrm{sep}}\right)$; the systolic stretch index $\left(\mathrm{SSI}_{\text {sep-lat }}\right)$; and the internal stretch index $\left(\mathrm{ISF}_{\text {sep-lat }}\right)$. Lastly, septal strain patterns were visually classified to the following pre-specified patterns: double peaked systolic shortening (LBBB-1); early preejection shortening followed by prominent systolic stretch (LBBB-2); and pseudonormal shortening with a late-systolic shortening peak and less pronounced endsystolic stretch (LBBB-3).

\section{Electrocardiographic and echocardiographic parameters}

A detailed description of the ECG- and echocardiographic analysis has been published previously [10]. In brief, a baseline 12-lead ECG was recorded for QRS duration measurements and QRS morphology assessment. Subsequently, a $3 \mathrm{D}$ vector loop was constructed from the 12-lead ECG to calculate the $\mathrm{QRS}_{\mathrm{AREA}}$. The presence of 
apical rocking was assessed during baseline echocardiography, and defined as a short systolic septal-to-lateral rocking motion of the apex [13]. In addition, the interventricular mechanical delay (IVMD) was measured as the timing difference between LV and RV pre-ejection intervals. Subsequently, the CRT-Age-Vectorcardio-

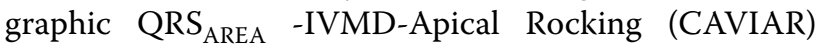
score was calculated as previously described in the MARC main study [10].

\section{Statistical analysis}

Statistical analysis was performed in the study core lab (University Medical Center Groningen, Groningen, The Netherlands) by a specialized team led by a bioinformatician (BG) using the R software (R Foundation for Statistical Computing, Vienna, Austria). Continuous variables are expressed as mean \pm standard deviation or in absence of a normal distribution as median and interquartile range. Categorical variables are presented as numbers and percentages. Strain parameters were compared between CRT responder and non-responder groups by an independent student t-test, or a non-parametric test when appropriate. Correlations between strain parameters and echocardiographic CRT response were assessed using the Pearson's correlation coefficient or when normal distribution was absent, the Spearman's Rho correlation coefficient. Receiver operating characteristics (ROC) curve analysis was used to find optimal cut-off values and determine predictive value of strain parameters. Univariable linear regression analysis was performed to assess the association of other (clinical, CMR and CAVIAR) parameters with echocardiographic CRT response. To test the additional value of SLICE strain analysis on top of conventional determinants of CRT response, multivariable linear regression analysis was performed by entering the best performing strain parameter (based on R) to a model with standard CMR parameters (model 1), clinical parameters (model 2) and the CAVIAR score parameters (model 3). Multiple testing was corrected for when appropriate. A $p$-value of $<0.05$ was considered statistically significant.

\section{Results}

After screening 63 patients, three patients were excluded from the analysis due to incomplete- or insufficient CMR image quality. Of the remaining 60 patients, 57 patients completed the 1-year follow up. One patient was lost to follow-up because of non-cardiac death (lung carcinoma), one withdrew informed consent, and one lacked sufficient image-quality during echocardiographic examination. Patient characteristics of the remaining 57 patients are presented in Table 1. Mean LVESV change after one year was $-32 \pm 27 \%$ with 14 (25\%) patients showing less than $15 \%$ LVESV reduction (CRT non-responders).

\section{SLICE parameters and CRT response}

Strain values for the total patient group, and both responder and non-responder subgroups are presented in Additional file 1: Table S1 of the appendix. Basic strain values measured as peak strain in the septal and lateral wall region showed weak correlations with LVESV change as demonstrated in Table 2. In contrast, the

Table 1 Patient characteristics at baseline and twelve months follow-up

\begin{tabular}{|c|c|c|c|c|}
\hline Variable & Total group $(n=57)$ & Responders $(n=43)$ & Non-responders $(n=14)$ & $P$-value \\
\hline Age (years) & $65 \pm 10$ & $63 \pm 10$ & $71 \pm 9$ & 0.013 \\
\hline Gender ( $n, \%$ male) & $30(53 \%)$ & $20(47 \%)$ & $10(71 \%)$ & 0.132 \\
\hline Etiology (n, \% ICMP) & $13(23 \%)$ & $5(12 \%)$ & $8(57 \%)$ & 0.001 \\
\hline ECG-QRS width (ms) & $176(165-188)$ & $180(168-194)$ & $169(152-176)$ & 0.011 \\
\hline ECG-QRS morph (n, \% LBBB) & $45(79 \%)$ & $38(88 \%)$ & $7(50 \%)$ & 0.005 \\
\hline ECG-QRS area $(\mu \mathrm{Vs})$ & $138(118-159)$ & $143(126-168)$ & $115(78-126)$ & 0.002 \\
\hline Echo-IVMD (ms) & $50 \pm 29$ & $58 \pm 25$ & $24 \pm 24$ & $<0.001$ \\
\hline Echo-apical rocking (n, \%) & $39(68 \%)$ & $34(79 \%)$ & $5(36 \%)$ & 0.006 \\
\hline CAVIAR score (points) & $3 \pm 3$ & $4 \pm 2$ & $0 \pm 2$ & $<0.001$ \\
\hline CMR-LVEDV (ml) & $283(227-338)$ & $297(231-380)$ & $256(220-297)$ & 0.181 \\
\hline CMR-LVEF (\%) & $28 \pm 10$ & $27 \pm 10$ & $30 \pm 6$ & 0.092 \\
\hline CMR-Scar size (\% LV mass) & $1.3(0.0-5.7)$ & $0.0(0.0-2.7)$ & $5.7(0.0-15.1)$ & 0.028 \\
\hline Echo-Change in LVESV after 12 months (\%) & $-32 \pm 27$ & $-44 \pm 18$ & $4 \pm 15$ & $<0.001$ \\
\hline ECG-Change in QRS width after CRT (ms) & $-32 \pm 28$ & $-37 \pm 26$ & $-15 \pm 29$ & 0.017 \\
\hline
\end{tabular}

Continuous variables are expressed as mean \pm standard deviation or in absence of a normal distribution as median and interquartile range. Categorical variables are

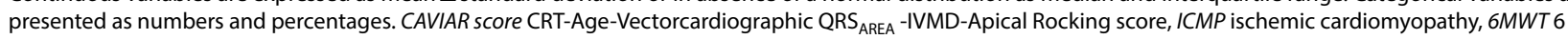
minute walk test, $L B B B$ left bundle branch block, IVMD interventricular mechanical delay, LVEDV left ventricular end-diastolic volume, $L V E F$ left ventricular ejection fraction, CMR cardiovascular magnetic resonance, Echo echocardiography, ECG electrocardiogram 
Table 2 Correlation of SLICE-derived strain parameters with CRT response (left ventricular end-systolic volume (LVESV) change after 12 months)

\begin{tabular}{llrr}
\hline & Variable & \multicolumn{1}{l}{ R } & p-value \\
\hline Basic strain parameters & Peak strain $_{\text {sep }}(\%)$ & -0.37 & 0.004 \\
& Peak strain $_{\text {lat }}(\%)$ & 0.33 & 0.012 \\
& ESS $_{\text {sep }}(\%)$ & -0.63 & $<0.001$ \\
& ESS $_{\text {lat }}(\%)$ & 0.44 & $<0.001$ \\
Timing parameters & Onset-delay (ms) & 0.06 & 0.669 \\
& Peak-delay (ms) & -0.22 & 0.098 \\
Strain pattern parameters & SRS $_{\text {sep }}(\%)$ & -0.56 & $<0.001$ \\
& SSI $_{\text {sep-lat }}(\%)$ & -0.53 & $<0.001$ \\
& ISF $_{\text {sep-lat }}$ & -0.58 & $<0.001$ \\
\hline
\end{tabular}

$R$ correlation coefficient with LVESV change after twelve months; Peak strain peak negative strain; ESS end-systolic strain; onset-delay septal to lateral delay in onset contraction; peak-delay septal to lateral time difference in peak shortening; $S R S_{\text {sep }}$ systolic rebound stretch of the septum; SSI $I_{\text {sep-lat }}$ systolic stretch index; $I S F_{\text {sep-lat }}$ internal stretch factor

basic strain parameter end-systolic septal strain $\left(\mathrm{ESS}_{\text {sep }}\right)$ showed the highest correlation coefficient of all parameters $(\mathrm{R}-0.63, p<0.001)$ with good predictive value for CRT response (AUC 0.81, $p<0.001$, Fig. 2) and an optimal cut-off of $0.3 \%$. Comparing subgroups by QRS morphology, correlation of ESS $_{\text {sep }}$ with CRT response was stronger within interventricular conduction delay (IVCD) patients $(\mathrm{R}-0.87, p<0.001)$ compared with LBBB patients $(\mathrm{R}-0.48, p=0.001)$. Timing parameters onset-delay and peak-delay were unrelated to LVESV change and yielded no predictive value for CRT response (Additional file 1: Table S2). On the other hand, strain pattern parameters all correlated with LVESV change $\left(\mathrm{SRS}_{\text {sep }} \mathrm{R}-0.56\right.$; $\mathrm{SSI}_{\text {sep-lat }} \mathrm{R}-0.53$; $\mathrm{ISF}_{\text {sep-lat; }} \mathrm{R}-0.58$, all $p<0.001)$. As demonstrated in Fig. 3, septal strain curves were visually classified as LBBB-1 pattern in $25 \%$, LBBB-2 in $44 \%$, and LBBB-3 in 32\% of the patients. Patients with pattern LBBB-1 or LBBB-2 demonstrated significantly more reverse remodeling compared to pattern LBBB-3. In addition, the response rate in the LBBB-1 and LBBB-2 was a two-fold higher than the response rate in the LBBB-3 group ( $88 \%$ / 93\% versus 44\%), see Additional file 1: Table S3. Comparing electrical parameters between the LBBB groups we found QRS morphology to be more frequently IVCD in LBBB-3 (44\%) compared to LBBB-1 $(29 \%)$ or LBBB-2 $(0 \%)$ patients $(\mathrm{p}=0.001$ for comparison). However, no differences in QRS duration were found between groups.

\section{Clinical parameters and CRT response}

CRT responders were younger, had relatively less often ischemic etiology and wider QRS duration compared to non-responders (Table 1). LV lead position was lateral in $67 \%$, posterolateral in $24 \%$ and anterolateral in $8 \%$ (left anterior oblique view) and basal in $20 \%$, mid in $61 \%$ and apical in 18\% (Right anterior oblizue view). LV lead location was congruent with scar location on LGE imaging in $18 \%$ of 47 patients. Patients with scar at LV lead location showed less LVESV reduction compared to others ( $-13 \pm 22 \%$ vs. $-36 \pm 24 \%$; $=0.008)$. The LV capture threshold was $1.2 \pm 0.7 \mathrm{~V}$ and was not related to presence of scar. Standard CMR parameters that correlated with reverse remodeling after CRT in univariable linear regression analysis included LV end-diastolic volume
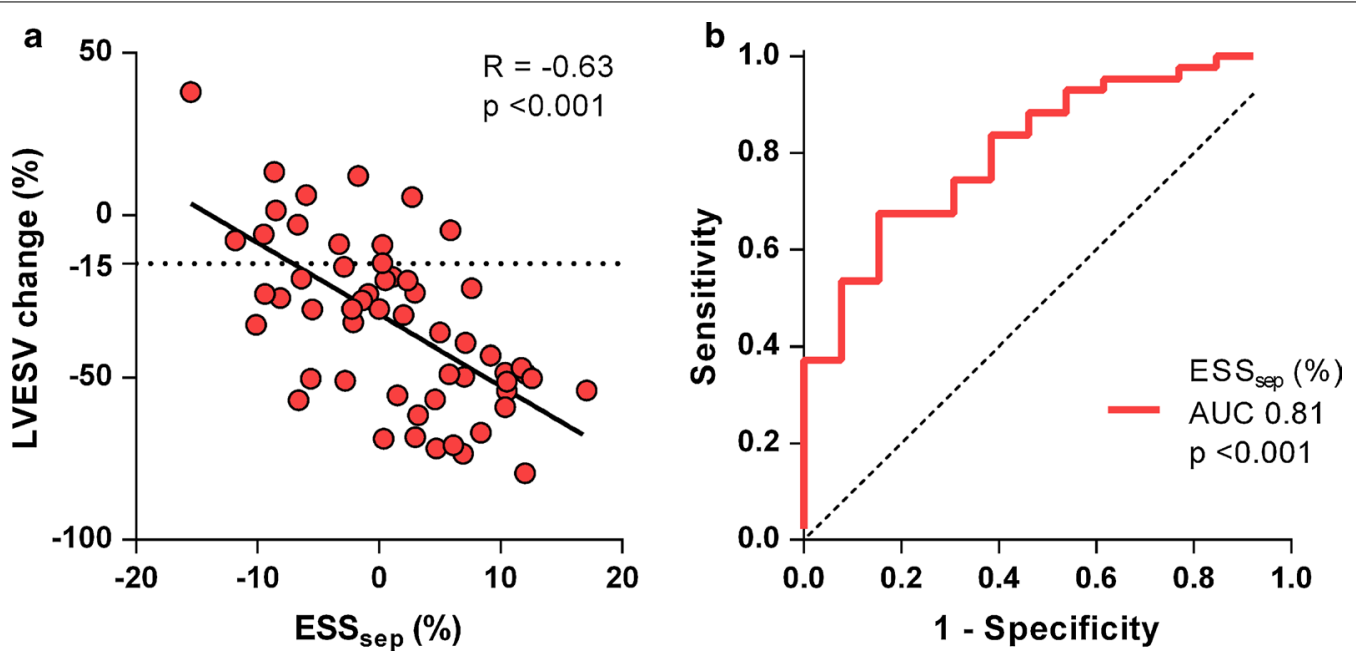

Fig. 2 End-systolic septal strain and reverse remodeling after cardiac resynchronization therapy (CRT). a The end-systolic septal strain (ESS

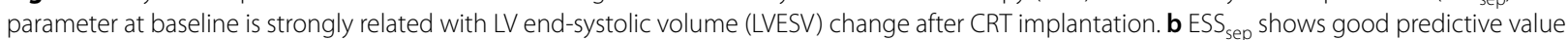
for CRT response ( $\geq 15 \%$ LVESV reduction) as demonstrated by receiver operating characteristic (ROC) curve analysis 

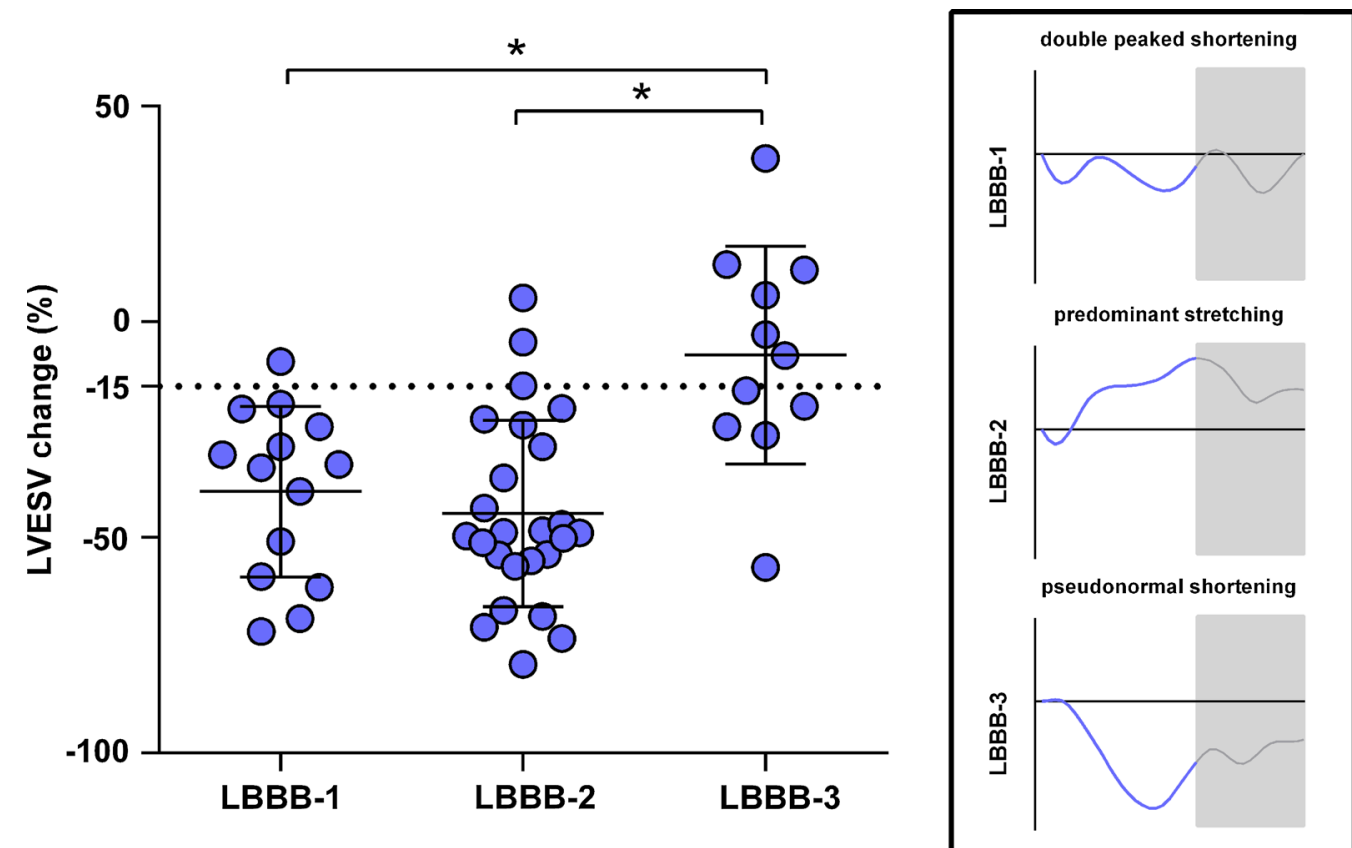

Fig. 3 Visual classification of septal strain patterns to estimate CRT response. Septal strain patterns are classified to pre-specified categories: double peaked shortening (LBBB-1), predominant stretching (LBBB-2) or pseudonormal shortening (LBBB-3). Statistical differences between LBBB categories are marked with an asterisk

Table 3 Linear regression analysis

\begin{tabular}{|c|c|c|c|c|c|c|}
\hline \multirow[b]{2}{*}{ CMR parameters / ESS $_{\text {sep }}$} & \multicolumn{3}{|c|}{ Univariable analysis } & \multicolumn{3}{|c|}{ Model 1} \\
\hline & Beta & $95 \% \mathrm{Cl}$ & $P$-value & Beta & $95 \% \mathrm{Cl}$ & $P$-value \\
\hline CMR-LVEDV (per 10 ml) & -0.80 & -1.53 to -0.07 & 0.028 & 0.03 & $-0.86-0.93$ & 0.938 \\
\hline CMR-LVEF (per \%) & 0.79 & $0.08-1.51$ & 0.029 & 0.42 & $-0.41-1.24$ & 0.312 \\
\hline CMR-Scar size (per \% LV mass) & 2.22 & $1.21-3.23$ & $<0.001$ & 1.32 & $-0.20-2.83$ & 0.088 \\
\hline Scar at LV lead location (yes) & 12.54 & $3.81-21.27$ & 0.006 & -4.07 & $-25.0-16.9$ & 0.696 \\
\hline CMR-ESS $S_{\text {sep }}($ per \%) & -2.20 & -2.94 to -1.47 & $<0.001$ & -1.25 & -2.29 to -0.21 & 0.019 \\
\hline \multicolumn{4}{|l|}{ Clinical parameters / ESS $_{\text {sep }}$} & \multicolumn{3}{|l|}{ Model 2} \\
\hline Age (per year) & 1.06 & $0.43-1.70$ & 0.001 & 0.68 & $0.17-1.20$ & 0.009 \\
\hline Gender (male) & 17.3 & $3.94-30.67$ & 0.011 & -1.24 & $-12.63-10.16$ & 0.831 \\
\hline Etiology (ICMP) & 30.89 & $16.19-45.58$ & $<0.001$ & 12.20 & $-1.68-26.08$ & 0.085 \\
\hline ECG-QRS width (per ms) & -0.29 & -0.55 to -0.02 & 0.033 & -0.08 & $-0.30-0.14$ & 0.481 \\
\hline ECG-QRS morph (LBBB) & -29.12 & -44.62 to -13.63 & $<0.001$ & -9.58 & $-23.80-4.63$ & 0.186 \\
\hline CMR-ESS & -2.20 & -2.94 to -1.47 & $<0.001$ & -1.32 & -2.18 to -0.47 & 0.002 \\
\hline \multicolumn{4}{|l|}{ CAVIAR components / ESS sep } & \multicolumn{3}{|c|}{ Model 3} \\
\hline Age (per year) & 1.06 & $0.43-1.70$ & 0.001 & 0.63 & $0.14-1.13$ & 0.013 \\
\hline ECG-QRS area (per $\mu \mathrm{Vs})$ & -0.32 & -0.45 to -0.19 & $<0.001$ & -0.18 & -0.30 to -0.05 & 0.005 \\
\hline Echo-IVMD (per ms) & -0.52 & -0.73 to -0.31 & $<0.001$ & 0.12 & $-0.35-0.10$ & 0.288 \\
\hline Echo-apical rocking & -26.08 & -39.59 to -12.56 & $<0.001$ & -2.66 & $-15.56-10.25$ & 0.687 \\
\hline $\mathrm{CMR} \mathrm{ESS}_{\text {sep }}($ per \%) & -2.20 & -2.94 to -1.47 & $<0.001$ & -1.51 & -2.35 to -0.67 & $<0.001$ \\
\hline
\end{tabular}

LVEDV left ventricular end-diastolic volume, LVESV left ventricular end-systolic volume, LVEF left ventricular ejection fraction, ESS ${ }_{\text {sep }}$ end-systolic septal strain, ICMP ischemic cardiomyopathy, CAVIAR score CRT-Age-Vectorcardiographic QRS $_{\text {AREA }}$-IVMD-Apical Rocking score, IVMD interventricular mechanical delay, LBBB-3 visual classification of strain pattern 3 
(LVEDV), LVEF, scar size and scar at LV lead location as demonstrated in Table 3. Addition of the best performing strain parameter, $\mathrm{ESS}_{\text {sep }}$, to standard CMR parameters in a multivariable model (model 1) showed ESS sep $_{\text {to be }}$ independently related to LVESV change whereas other CMR parameters were not, although there was a trend towards significance for scar size. Clinical parameters that were associated with CRT response included age, gender, etiology, QRS duration and QRS morphology. In a multivariable model with clinical determinants (model 2), ESS $_{\text {sep }}$ showed to be independently related to LVESV change together with age. The CAVIAR score parameters age, $\mathrm{QRS}_{\mathrm{AREA}}, \mathrm{IVMD}$, and apical rocking, were all significantly associated with CRT response. Addition of ESS $_{\text {sep }}$ to CAVIAR (model 3), showed ESS sep to be independently related to LVESV change together with age and QRS AREA $_{\text {. }}$

\section{Discussion}

This study is the first to demonstrate predictive value of the novel SLICE strain technique for functional LV recovery after CRT implantation. Various SLICE strain parameters showed to be closely related with CRT response after 1 year. When comparing different types of strain parameters, strain pattern- rather than timing variables correlated with CRT response. Of all strain parameters, end-systolic septal strain demonstrated the strongest correlation with LV reverse remodeling after 1 year. Moreover, multivariable regression analysis showed end-systolic septal strain to be additive to clinical (age) and electrical $\left(\mathrm{QRS}_{\mathrm{AREA}}\right)$ parameters in estimating potential benefit from CRT.

\section{Comparison of strain parameters}

In patients with LBBB, slow cell-to-cell LV conduction results in a time-difference of electrical activation between the septal and lateral wall. Although SLICE analysis revealed mechanical contraction always to be delayed in the lateral wall relative to the septal wall, absolute time delays between the septal and lateral wall (onset-delay; peak-delay) were unrelated to the amount of echocardiographic response after CRT. These findings are in agreement with those of the predictors of response to cardiac resynchronization therapy (PROSPECT) trial, showing disappointing results of timing (i.e. dyssynchrony) parameters [14]. More recent studies suggest strain pattern- (i.e. discoordination) rather than timing parameters to be related to CRT response $[3,4,6]$. In contrast to timing parameters, strain pattern parameters incorporate regional function by measuring myocardial contraction and stretching in percentage strain units. In line with previous reports, strain pattern parameters $\left(\mathrm{SRS}_{\text {sep }}\right.$; $\mathrm{SSI}_{\text {sep-lat }} \mathrm{ISF}_{\text {sep-lat }}$ ) all correlated with reverse remodeling after CRT and accurately predicted response to CRT [3, $4,6]$.

\section{Septal strain analysis}

During LBBB, systolic stretching is most profound in early-activated (i.e. septal) segments whereas contractile function is increased in late-activated (i.e. lateral wall) regions, resulting in an imbalanced septal-to-lateral work load ratio [15-17]. CRT subsequently recruits myocardial work from the septum, leading to a homogenized work distribution and improving cardiac pump efficiency [16-19]. In the present study, we found end-systolic strain of the septum (ESS ${ }_{\text {sep }}$ ) to be strongest related with reverse remodeling after CRT as illustrated in Fig. 2. Previously, we performed a systematic comparison of strain parameters using multiple strain imaging techniques (CMR-TAG; CMR-FT; STE) and found ESS sep $_{\text {to }}$ be the best performing strain parameter in the prediction of CRT outcome irrespective of imaging technique [20]. The present study adds to the accumulating evidence that discoordination of the septum forms the mechanical substrate for functional LV improvement during electrical resynchronization. ESS ${ }_{\text {sep }}$ reflects net septum length change throughout systole and varied widely between patients ranging between - 15.5\% (shortening) and $17.1 \%$ (stretching). These large differences in strain can be easily detected by SLICE and require septal analysis in only two (end-diastolic and end-systolic) frames (Fig. 1a). Time duration of the specific SLICE-ESS sep $_{\text {measurement }}$ was only $12 \pm 2 \mathrm{~min}$. Previously, our validation study showed high agreement of SLICE-ESS ${ }_{\text {sep }}$ with gold standard CMR-TAG (ICC: 0.76) with excellent intra-observer reproducibility (ICC: 0.94) and good inter-observer reproducibility (ICC: 0.86) [9]. Reproducibility of SLICEESS $_{\text {sep }}$ was higher compared to other strain parameters, presumably because of the wide spread in strain values. ROC curve analysis in the present study revealed an optimal cut-off value to predict CRT response around zero (0.3) percent. Patients with septal stretching rather than shortening during systole (positive numbers indicate stretching) are highly inefficient at baseline and leave more room for improvement in contractile function after CRT. These patients showed a three-fold larger reduction in LVESV compared to patients with preserved septal contraction. Alternatively, septal behavior can be evaluated in a subjective manner by the visual classification of septal strain curves to a pre-described pattern. Patients with a typical LBBB (i.e. LBBB-1 or LBBB-2) pattern demonstrated larger reductions in LVESV compared to patients with a pseudo-normal (i.e. LBBB-3) which is in line with previous studies $[5,8,21]$. However, calculation of quantitative strain parameters may be preferable as they are less dependent on the observer's interpretation 
compared to the subjective classification of septal strain patterns.

\section{The role of SLICE strain analysis in clinical practice}

In clinical practice, the role of CMR is of interest as it offers accurate LVEF measurement combined with LGE imaging to guide LV lead placement in CRT candidates [22-26]. Leyva et al. demonstrated LV lead deployment away from scarred myocardium to result in better clinical outcome while pacing in scarred myocardium was associated with the worst outcome [27]. In the present study, the role of SLICE strain analysis was compared to other CMR parameters to determine its value on top of standard information provided by CMR examination (model 1). Multivariable modeling showed ESS sep to be the only parameter independently related to LVESV change, although there was a trend towards significance for scar size. Scar size and septal discoordination were interrelated $(\mathrm{R}=-0.51 ; \mathrm{p}<0.001)$ as they possibly share mutual information on LV contractility [5]. Recent animal experiments showed that decreasing LV contractility by creating myocardial infarctions resulted in less septal stretching [28]. Information on global scarring is therefore partially incorporated in ESS with lower values indicating poor CRT outcome.

Furthermore, the role of SLICE in relation to clinical parameters was explored. Clinical predictors of CRT response included age, gender, etiology, QRS width and QRS morphology (see Fig. 4), all considered to be traditional determinants of CRT response [1, 29]. The SLICE- ESS $_{\text {sep }}$ parameter may add to the decision for CRT, especially in patients with IVCD in whom benefit from CRT is doubted. It can be appreciated in Fig. 4 that there is large variation in response among

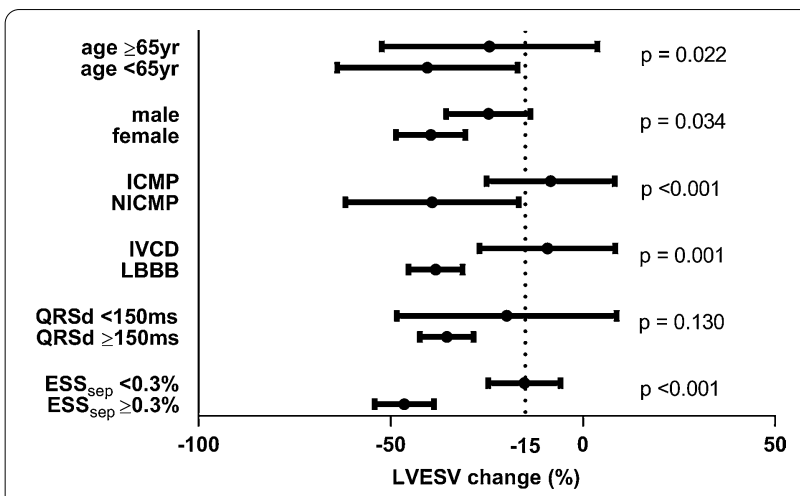

Fig. 4 Clinical factors and ESS ${ }_{\text {sep }}$ influencing the likelihood of response to CRT. The magnitude of benefit from CRT is demonstrated for different subgroups divided by age; gender; etiology; QRS morphology; QRSd; and ESS sep. Compared to clinical factors, SLICE- ESS $_{\text {sep }}$ demonstrates relatively large discriminative power
IVCD patients (ranging from non-response to superresponse). Whereas strict $\mathrm{LBBB}$ is usually accompanied by septal discoordination (resulting in CRT response), this is much less certain in patients with IVCD. It could be hypothesized that electro-mechanical dissociation plays a larger role in IVCD than LBBB patients. Additional SLICE analysis may be used to confirm "true LBBB" septal discoordination in these patients. We found $\mathrm{ESS}_{\text {sep }}$ to provide predictive value over QRS morphology (model 2). Moreover, predictive performance of ESS ${ }_{\text {sep }}$ was stronger within IVCD patients than LBBB patients. The potential value of ESS sep $_{\text {in IVCD patients }}$ is illustrated by two examples in Fig. 5. It should be noted, however, that IVCD patients composed of only $21 \%$ of the study population.

Lastly, the value of SLICE was evaluated with respect to a specially designed prediction model in the MARC main study (model 3). The CAVIAR response score incorporates age, $\mathrm{QRS}_{\mathrm{AREA}}$ derived by vector-loop ECG analysis and two echocardiographic parameters being IVMD and detection of apical rocking [10]. Addition of ESS to CAVIAR in multivariable analysis showed ESS sep $_{\text {to }}$ be an independent predictor of response together with age and QRS $_{\text {AREA }}$, whereas echocardiographic parameters (IVMD; apical rocking) were expelled from the model. These findings indicate that response to CRT is multifactorial [2], and that combining clinical information (age) together with electrocardiographic $\left(\mathrm{QRS}_{\mathrm{AREA}}\right)$ and

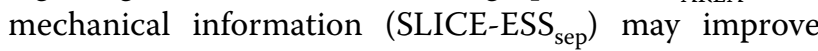
patient selection for CRT. As QRS AREA $_{\text {can be measured }}$ from a standard 12-lead ECG and SLICE-ESS sep from standard CMR cine images, the work-up of CRT candidates is feasible in clinical practice.

\section{Limitations}

Some limitations need to be recognized. First, this substudy of the MARC involves a subset of patients who underwent additional CMR examination. Although patient characteristics were comparable with the overall MARC population, this may have introduced some selection bias. As a next step, predictive performance of SLICE-ESS $_{\text {sep }}$ should be evaluated in a validation cohort which is part of future work. Secondly, $18 \%$ of patients had their LV lead implanted in a region with myocardial scarring. Targeting LV lead placement outside scar regions could potentially improve response to CRT and may impact predictive value of ESS $_{\text {sep }}$ albeit not being investigated in the present study. Thirdly, CMR feature tracking (CMR-FT) post-processing software is now commercially available and offers automated strain analysis on standard CMR cine images. Nevertheless, CMRFT has several downsides as it requires the purchase of commercial software and the user is not able to track and 

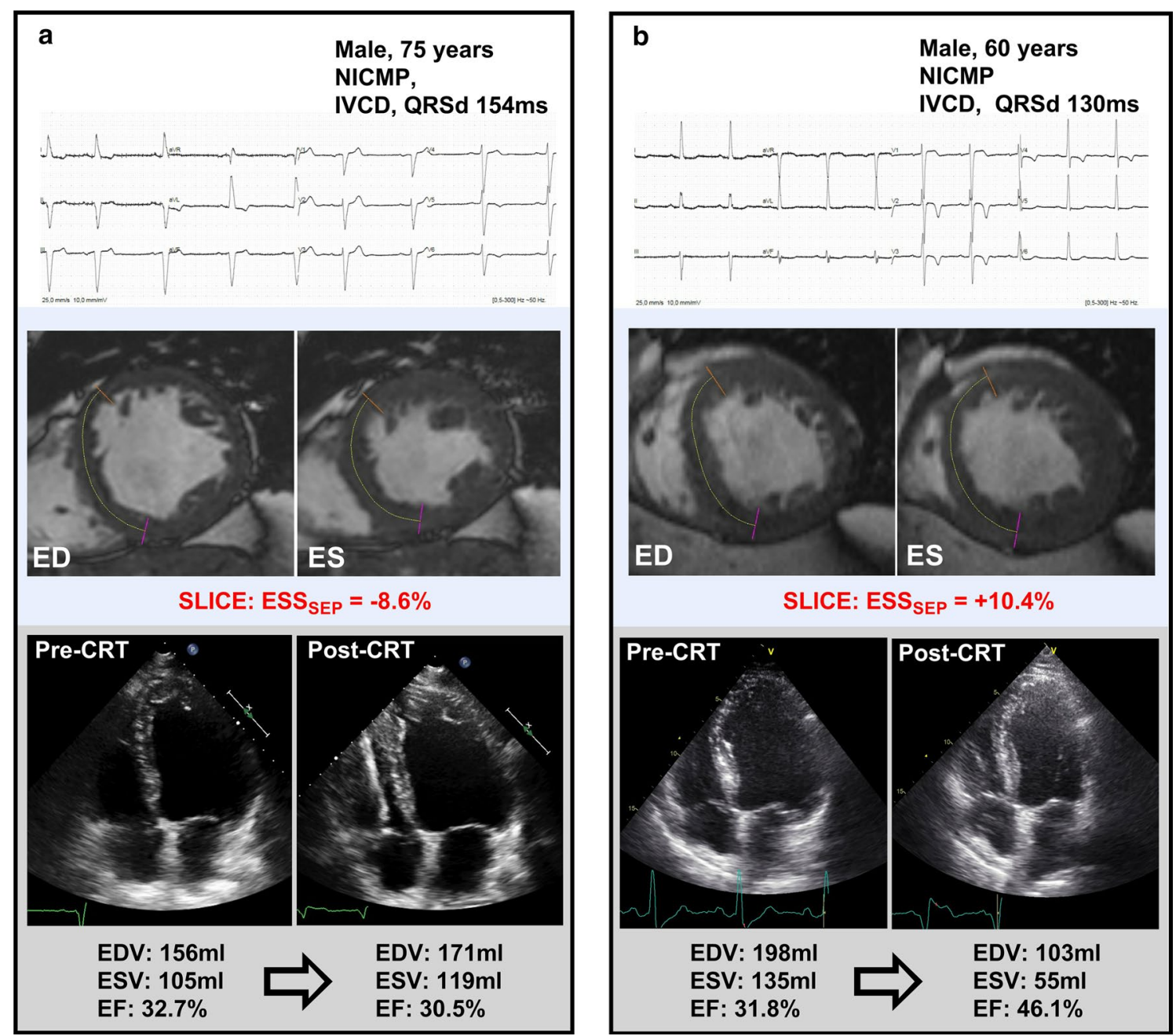

Fig. 5 Two typical examples demonstrating the additional value of SLICE strain analysis in clinical practice. a Typical example of a grey-zone CRT candidate with mixed favorable (non-ischemic cardiomyopathy (NICMP); QRSd $\geq 150 \mathrm{~ms}$ ), and unfavorable (male; age $\geq 65$ years; IVCD)

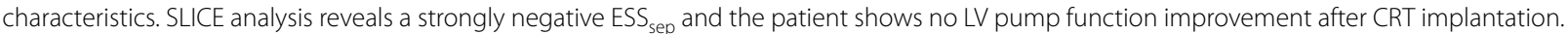
b Another patient with mixed favorable (NICMP; age < 65 years), and unfavorable (male; IVCD; QRSd < 150 ms) characteristics. This patient, however, demonstrates a strongly positive $\mathrm{ESS}_{\text {sep }}$ and becomes a CRT super-responder

trace the analysis steps. SLICE, on the other hand, can be performed without the use of specialized software tools. Fourthly, strain parameters that require SLICE analysis of the entire strain curve may take a long processing time (up to $60 \mathrm{~min}$ ). Measuring the specific ESS sep $_{\text {parameter, }}$ however, requires only two SLICE measurements and can be performed in around twelve minutes. Furthermore, the SLICE analysis could be standardized by implementing radial taglines to standard cine imaging (Additional file 1: Figure S2). Lastly, SLICE relies on strain measures from in-plane motion of anatomic landmarks. However, the apparent in-plane movements may also be caused by through-plane displacements of oblique or tapering structures that form the anatomic landmarks. For this reason, we could only analyze the mid-LV slice, since this plane is relatively motion independent. Nevertheless, variation in strain values between basal, mid- and apical LV segments are relatively small [30].

\section{Conclusions}

The practicable SLICE strain technique helps the clinician to estimate potential benefit from CRT by analyzing standard CMR cine images without the need for commercial software. Of all strain parameters, end-systolic septal strain $\left(E_{\text {sep }}\right)$ demonstrated the strongest correlation with reverse remodeling after CRT. This parameter can be measured in around 12 min and may be of special interest in patients with non-strict LBBB morphology 
in whom CRT benefit is doubted. New clinical trials are needed to determine whether detection of septal discoordination yields additive value to traditional CRT parameters in clinical practice.

\section{Supplementary Information}

The online version contains supplementary material available at https://doi. org/10.1186/s12968-020-00701-4.

Additional file 1: Table S1. Comparison of strain parameters between CRT responders and non-responders. Table $\mathbf{S 2}$. Predictive value of strain parameters for CRT response ( $\geq 15 \%$ reduction in LVESV). Table S3. Septal strain patterns and CRT response. Figure S1. Localization of the anatomical landmarks. Figure S2. Modification of the SLICE technique by implementing radial taglines.

\section{Abbreviations}

AV: Atrioventricular; AVC: Aortic valve closure; bSSFP: Balanced steady state free precession; CAVIAR: CRT-age-vectorcardiographic QRSarea-RVMDapical rocking score; CMR: Cardiovascular magnetic resonance; CRT: Cardiac resynchronization therapy; ECG: Electrocardiogram; ED: End-diastole; ESS:

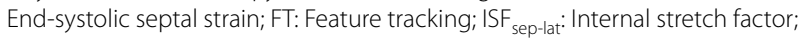
IVCD: Intraventricular conduction delay; IVMD: Interventricular mechanical delay; LBBB: Left bundle branch block; LGE: Late gadolinium enhancement; LV: Left ventricle/left ventricular; LVEDV: Left ventricular end-diastolic volume; LVEF: Left ventricular ejection fraction; LVESV: Left ventricular end-systolic volume; MARC: Markers and Response to CRT study; RV: Right ventricle/right ventricular; SLICE: Segment length in cine; SRS: Systolic rebound stretch; SSI: Systolic stretch index; TAG: Tagging; TE: Echo time; TR: Repetition time; V: Interventricular.

\section{Acknowledgements}

None.

\section{Authors' contributions}

AZ made contributions to the conception; design of the work; the acquisition, analysis; interpretation of data; drafted the work or substantively revised it; approved the submitted version (and any substantially modified version that involves the author's contribution to the study); agreed both to be personally accountable for the author's own contributions and to ensure that questions related to the accuracy or integrity of any part of the work, even ones in which the author was not personally involved, are appropriately investigated, resolved, and the resolution documented in the literature. RN made contributions to the interpretation of data; drafted the work or substantively revised it; approved the submitted version (and any substantially modified version that involves the author's contribution to the study); agreed both to be personally accountable for the author's own contributions and to ensure that questions related to the accuracy or integrity of any part of the work, even ones in which the author was not personally involved, are appropriately investigated, resolved, and the resolution documented in the literature. NJB made contributions to the analysis; interpretation of data; drafted the work or substantively revised it; approved the submitted version (and any substantially modified version that involves the author's contribution to the study); agreed both to be personally accountable for the author's own contributions and to ensure that questions related to the accuracy or integrity of any part of the work, even ones in which the author was not personally involved, are appropriately investigated, resolved, and the resolution documented in the literature. AHM made contributions to the interpretation of data; drafted the work or substantively revised it; approved the submitted version (and any substantially modified version that involves the author's contribution to the study); agreed both to be personally accountable for the author's own contributions and to ensure that questions related to the accuracy or integrity of any part of the work, even ones in which the author was not personally involved, are appropriately investigated, resolved, and the resolution documented in the literature. KV made contributions to the interpretation of data; drafted the work or substantively revised it; approved the submitted version (and any substantially modified version that involves the author's contribution to the study); agreed both to be personally accountable for the author's own contributions and to ensure that questions related to the accuracy or integrity of any part of the work, even ones in which the author was not personally involved, are appropriately investigated, resolved, and the resolution documented in the literature. FJ made contributions to the interpretation of data; drafted the work or substantively revised it; approved the submitted version (and any substantially modified version that involves the author's contribution to the study); agreed both to be personally accountable for the author's own contributions and to ensure that questions related to the accuracy or integrity of any part of the work, even ones in which the author was not personally involved, are appropriately investigated, resolved, and the resolution documented in the literature. MM made contributions to the interpretation of data; drafted the work or substantively revised it; approved the submitted version (and any substantially modified version that involves the author's contribution to the study); agreed both to be personally accountable for the author's own contributions and to ensure that questions related to the accuracy or integrity of any part of the work, even ones in which the author was not personally involved, are appropriately investigated, resolved, and the resolution documented in the literature. BG made contributions to the interpretation of data; drafted the work or substantively revised it; approved the submitted version (and any substantially modified version that involves the author's contribution to the study); agreed both to be personally accountable for the author's own contributions and to ensure that questions related to the accuracy or integrity of any part of the work, even ones in which the author was not personally involved, are appropriately investigated, resolved, and the resolution documented in the literature. MR made contributions to the interpretation of data; drafted the work or substantively revised it; approved the submitted version (and any substantially modified version that involves the author's contribution to the study); agreed both to be personally accountable for the author's own contributions and to ensure that questions related to the accuracy or integrity of any part of the work, even ones in which the author was not personally involved, are appropriately investigated, resolved, and the resolution documented in the literature. ICG made contributions to the conception; design of the work; interpretation of data; drafted the work or substantively revised it; approved the submitted version (and any substantially modified version that involves the author's contribution to the study); agreed both to be personally accountable for the author's own contributions and to ensure that questions related to the accuracy or integrity of any part of the work, even ones in which the author was not personally involved, are appropriately investigated, resolved, and the resolution documented in the literature. MAV made contributions to the interpretation of data; drafted the work or substantively revised it; approved the submitted version (and any substantially modified version that involves the author's contribution to the study); agreed both to be personally accountable for the author's own contributions and to ensure that questions related to the accuracy or integrity of any part of the work, even ones in which the author was not personally involved, are appropriately investigated, resolved, and the resolution documented in the literature. ACR made contributions to the interpretation of data; drafted the work or substantively revised it; approved the submitted version (and any substantially modified version that involves the author's contribution to the study); agreed both to be personally accountable for the author's own contributions and to ensure that questions related to the accuracy or integrity of any part of the work, even ones in which the author was not personally involved, are appropriately investigated, resolved, and the resolution documented in the literature. CPA made contributions to the conception; design of the work; the acquisition, analysis; interpretation of data; drafted the work or substantively revised it; approved the submitted version (and any substantially modified version that involves the author's contribution to the study); agreed both to be personally accountable for the author's own contributions and to ensure that questions related to the accuracy or integrity of any part of the work, even ones in which the author was not personally involved, are appropriately investigated, resolved, and the resolution documented in the literature. All authors read and approved the final manuscript.

\section{Funding}

This research was funded within the framework of CTMM, the Centre for Translational Molecular Medicine (www.ctmm.nl), project COHFAR (Grant 01C-203), and supported by the Dutch Heart Foundation. 


\section{Availability of data and materials}

The datasets generated and/or analysed during the current study are not publicly available due to planned future publications.

\section{Ethics approval and consent to participate}

All subjects gave written informed consent and the local medical ethics committee (Amsterdam University Medical Centers, location VU medical center) approved data collection and management.

\section{Consent for publication}

Not applicable.

\section{Competing interests}

Dr. Vernooy received consultancy fee from Medtronic; research grants from Medtronic; speaker fees from St. Jude Medical. Dr. Maass received lecture fees from Medtronic and LivaNova. Dr. Vos received funding from CTMM COHFAR, CVON Predict, EU TrigTreat, EU CERT-ICD and GiLead to perform (pre)clinical studies. Dr. Allaart received speaker fees from Biotronik. All remaining authors declare that they have no conflict of interests.

\section{Author details}

${ }^{1}$ Department of Cardiology, Amsterdam Cardiovascular Sciences (ACS), Amsterdam University Medical Centers (AUMC), Location VU University Medical Center, De Boelelaan 1118, 1081 HV Amsterdam, The Netherlands. 2 Department of Cardiology, Radboud University Medical Center, Nijmegen, The Netherlands. ${ }^{3}$ Department of Cardiology, Thoraxcentre, University of Groningen, University Medical Centre Groningen, Groningen, The Netherlands. ${ }^{4}$ Department of Cardiology, Maastricht University Medical Centre, Maastricht, The Netherlands. ${ }^{5}$ Department of Cardiology, Amsterdam University Medical Centers (AUMC), Location Academic Medical Center, Amsterdam, The Netherlands. ${ }^{6}$ Department of Cardiology, University Medical Centre Utrecht, Utrecht, The Netherlands. ${ }^{7}$ Department of Medical Physiology, University of Utrecht, Utrecht, The Netherlands.

Received: 31 December 2019 Accepted: 24 August 2020

Published online: 11 January 2021

\section{References}

1. Brignole M, Auricchio A, Baron-Esquivias G, Bordachar P, Boriani G, Breithardt OA, et al. ESC guidelines on cardiac pacing and cardiac resynchronization therapy: the task force on cardiac pacing and resynchronization therapy of the European Society of Cardiology (ESC). Developed in collaboration with the European Heart Rhythm Association (EHRA). Europace. 2013:15(8):1070-118.

2. Daubert C, Behar N, Martins RP, Mabo P, Leclercq C. Avoiding nonresponders to cardiac resynchronization therapy: a practical guide. Eur Heart J. 2017;38(19):1463-72.

3. De Boeck BWL, Teske AJ, Meine M, Leenders GE, Cramer MJ, Prinzen FW, et al. Septal rebound stretch reflects the functional substrate to cardiac resynchronization therapy and predicts volumetric and neurohormonal response. Eur J Heart Fail. 2009;11(9):863-71.

4. Kirn B, Jansen A, Bracke F, van Gelder B, Arts T, Prinzen FW. Mechanical discoordination rather than dyssynchrony predicts reverse remodeling upon cardiac resynchronization. Am J Physiol Heart Circul Physiol. 2008:295(2):H640-6.

5. Leenders GE, Lumens J, Cramer MJ, De Boeck BW, Doevendans PA, Delhaas T, et al. Septal deformation patterns delineate mechanical dyssynchrony and regional differences in contractility: analysis of patient data using a computer model. Circ Heart Fail. 2012;5(1):87-96.

6. Lumens J, Tayal B, Walmsley J, Delgado-Montero A, Huntjens PR, Schwartzman D, et al. Differentiating electromechanical from nonelectrical substrates of mechanical discoordination to identify responders to cardiac resynchronization therapy. Circ Cardiovasc Imaging 2015:8(9):e003744.

7. van Everdingen WM, Zweerink A, Nijveldt R, Salden OAE, Meine M, Maass $\mathrm{AH}$, et al. Comparison of strain imaging techniques in CRT candidates: CMR tagging, CMR feature tracking and speckle tracking echocardiography. Int J Cardiovasc Imaging. 2018;34(3):443-56.
8. Risum N, Tayal B, Hansen TF, Bruun NE, Jensen MT, Lauridsen TK, et al. Identification of typical left bundle branch block contraction by strain echocardiography is additive to electrocardiography in prediction of long-term outcome after cardiac resynchronization therapy. J Am Coll Cardiol. 2015;66(6):631-41.

9. Zweerink A, Allaart CP, Kuijer JPA, Wu L, Beek AM, van de Ven PM, et al. Strain analysis in CRT candidates using the novel segment length in cine (SLICE) post-processing technique on standard CMR cine images. Eur Radiol. 2017:27(12):5158-68.

10. Maass AH, Vernooy K, Wijers SC, van't Sant J, Cramer MJ, Meine M, et al. Refining success of cardiac resynchronization therapy using a simple score predicting the amount of reverse ventricular remodelling: results from the markers and response to CRT (MARC) study. Europace. 2018;20(2):e1-10.

11. European Heart Rhythm A, European Society of C, Heart Rhythm S, Heart Failure Society of A, American Society of E, American Heart A, et al. 2012 EHRA/HRS expert consensus statement on cardiac resynchronization therapy in heart failure: implant and follow-up recommendations and management. Heart Rhythm. 2012;9(9):1524-76.

12. Flett AS, Hasleton J, Cook C, Hausenloy D, Quarta G, Ariti C, et al. Evaluation of techniques for the quantification of myocardial scar of differing etiology using cardiac magnetic resonance. JACC Cardiovasc Imaging. 2011;4(2):150-6.

13. Szulik M, Tillekaerts M, Vangeel V, Ganame J, Willems R, Lenarczyk R, et al. Assessment of apical rocking: a new, integrative approach for selection of candidates for cardiac resynchronization therapy. Eur J Echocardiogr. 2010;11(10):863-9.

14. Chung ES, Leon AR, Tavazzi L, Sun JP, Nihoyannopoulos P, Merlino J, et al. Results of the predictors of response to CRT (PROSPECT) trial. Circulation. 2008;117(20):2608-16.

15. Zweerink A, de Roest GJ, Wu L, Nijveldt R, de Cock CC, van Rossum AC, et al. Prediction of acute response to cardiac resynchronization therapy by means of the misbalance in regional left ventricular myocardial work. J Card Fail. 2016;22(2):133-42.

16. Prinzen FW, Vernooy K, De Boeck BW, Delhaas T. Mechano-energetics of the asynchronous and resynchronized heart. Heart Fail Rev. 2011;16(3):215-24.

17. Russell K, Eriksen M, Aaberge L, Wilhelmsen N, Skulstad H, Gjesdal O, et al. Assessment of wasted myocardial work: a novel method to quantify energy loss due to uncoordinated left ventricular contractions. Am J Physiol Heart Circ Physiol. 2013;305(7):H996-1003.

18. Cvijic M, Duchenne J, Unlu S, Michalski B, Aarones M, Winter S, et al. Timing of myocardial shortening determines left ventricular regional myocardial work and regional remodelling in hearts with conduction delays. Eur Heart J Cardiovasc Imaging. 2018;19(8):941-9.

19. Vernooy K, Cornelussen RN, Verbeek XA, Vanagt WY, van Hunnik A, Kuiper $M$, et al. Cardiac resynchronization therapy cures dyssynchronopathy in canine left bundle-branch block hearts. Eur Heart J. 2007;28(17):2148-55.

20. Zweerink A, van Everdingen WM, Nijveldt R, Salden OAE, Meine M, Maass $\mathrm{AH}$, et al. Strain imaging to predict response to cardiac resynchronization therapy: a systematic comparison of strain parameters using multiple imaging techniques. ESC Heart Fail. 2018;5(6):1130-40.

21. Jackson T, Sohal M, Chen Z, Child N, Sammut E, Behar J, et al. A U-shaped type II contraction pattern in patients with strict left bundle branch block predicts super-response to cardiac resynchronization therapy. Heart Rhythm. 2014;11(10):1790-7.

22. Bertini M, Mele D, Malagu M, Fiorencis A, Toselli T, Casadei F, et al. Cardiac resynchronization therapy guided by multimodality cardiac imaging. Eur J Heart Fail. 2016;18(11):1375-82.

23. Sommer A, Kronborg MB, Norgaard BL, Poulsen SH, Bouchelouche K, Bottcher M, et al. Multimodality imaging-guided left ventricular lead placement in cardiac resynchronization therapy: a randomized controlled trial. Eur J Heart Fail. 2016;18(11):1365-74.

24. Bilchick KC, Dimaano V, Wu KC, Helm RH, Weiss RG, Lima JA, et al. Cardiac magnetic resonance assessment of dyssynchrony and myocardial scar predicts function class improvement following cardiac resynchronization therapy. JACC Cardiovasc Imaging. 2008;1(5):561-8.

25. Leyva F. The role of cardiovascular magnetic resonance in cardiac resynchronization therapy. Heart Fail Clin. 2017;13(1):63-77.

26. Romano S, Judd RM, Kim RJ, Kim HW, Klem I, Heitner JF, et al. Featuretracking global longitudinal strain predicts death in a multicenter 
population of patients with ischemic and nonischemic dilated cardiomyopathy incremental to ejection fraction and late gadolinium enhancement. JACC Cardiovasc Imaging. 2018;11(10):1419-29.

27. Leyva F, Foley PW, Chalil S, Ratib K, Smith RE, Prinzen F, et al. Cardiac resynchronization therapy guided by late gadolinium-enhancement cardiovascular magnetic resonance. J Cardiovasc Magn Reson. 2011;13:29.

28. Aalen JM, Remme EW, Larsen CK, Andersen OS, Krogh M, Duchenne J, et al. Mechanism of abnormal septal motion in left bundle branch block: role of left ventricular wall interactions and myocardial scar. JACC Cardiovasc Imaging. 2019;12(12):2402-13.

29. Hsu JC, Solomon SD, Bourgoun M, McNitt S, Goldenberg I, Klein H, et al. Predictors of super-response to cardiac resynchronization therapy and associated improvement in clinical outcome: the MADIT-CRT (multicenter automatic defibrillator implantation trial with cardiac resynchronization therapy) study. J Am Coll Cardiol. 2012;59(25):2366-73.

30. Han Y, Chan J, Haber I, Peters DC, Zimetbaum PJ, Manning WJ, et al. Circumferential myocardial strain in cardiomyopathy with and without left bundle branch block. J Cardiovasc Magn Reson. 2010;12:2.

\section{Publisher's Note}

Springer Nature remains neutral with regard to jurisdictional claims in published maps and institutional affiliations.
Ready to submit your research? Choose BMC and benefit from:

- fast, convenient online submission

- thorough peer review by experienced researchers in your field

- rapid publication on acceptance

- support for research data, including large and complex data types

- gold Open Access which fosters wider collaboration and increased citations

- maximum visibility for your research: over 100M website views per year

At BMC, research is always in progress.

Learn more biomedcentral.com/submissions 